\title{
Synthesis of Poly-Ubiquitin Chains Using a Bifunctional Ubiquitin
} Monomer

Noort, G.J.V. van; Kooij, R.; Elliott, P.R.; Komander, D.; Ovaa, H.

\section{Citation}

Noort, G. J. V. van, Kooij, R., Elliott, P. R., Komander, D., \& Ovaa, H. (2017). Synthesis of PolyUbiquitin Chains Using a Bifunctional Ubiquitin Monomer. Organic Letters, 19(24), 6490-6493. doi:10.1021/acs.orglett.7b03085

Version: $\quad$ Not Applicable (or Unknown)

License: $\quad$ Leiden University Non-exclusive license

Downloaded from: https://hdl.handle.net/1887/116314

Note: To cite this publication please use the final published version (if applicable). 


\title{
Synthesis of Poly-Ubiquitin Chains Using a Bifunctional Ubiquitin Monomer
}

\author{
Gerbrand J. van der Heden van Noort, ${ }^{*},, \S \odot$ Raymond Kooij, ${ }^{\dagger, \S}$ Paul R. Elliott, ${ }^{\dagger}$ David Komander, ${ }^{\dagger}$ \\ and Huib Ovaa*, ${ }^{\dagger}$
}

\begin{abstract}
${ }^{\dagger}$ Department of Chemical Immunology, Leiden University Medical Centre, 2333 ZC Leiden, The Netherlands
${ }^{\ddagger}$ Medical Research Council Laboratory of Molecular Biology, Francis Crick Avenue, Cambridge Biomedical Campus, Cambridge CB2 $0 \mathrm{QH}$, United Kingdom
\end{abstract}

\section{Supporting Information}

ABSTRACT: An optimized large scale and highly reproducible route to orthogonally protected $\gamma$-thiolysine is reported. Its utility in the synthesis of bifunctional ubiquitin monomers is demonstrated. These ubiquitin synthons are employed in polymerization reactions giving access to synthetic polyubiquitin chains of defined linkage.
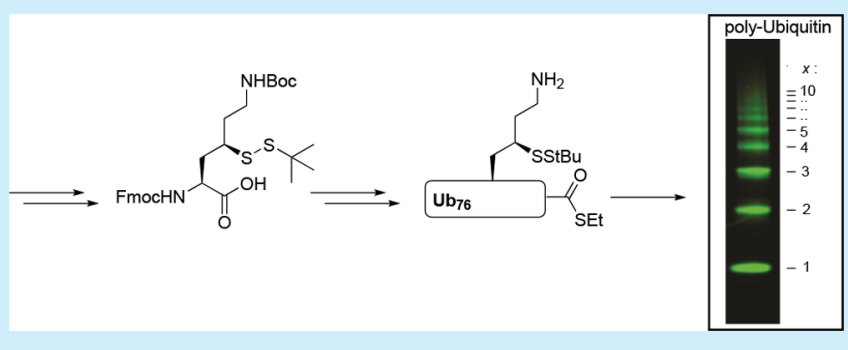

$\mathrm{U}$ biquitination of target proteins is a post-translational modification involved in almost all aspects of eukaryotic biology including the regulation of immune responses and cell cycle progression. ${ }^{1}$ The attachment of the 76 amino acid long protein ubiquitin $(\mathrm{Ub})$ to a nucleophilic functionality in the amino acid side chain of proteins alters the fate of the modified protein. The distinct signals that are brought about are mostly invoked by poly-ubiquitination, since $\mathrm{Ub}$ has the ability to form polymers by forming isopeptide bonds between the $\mathrm{N}$-terminal amine or any of the internal seven lysine $\varepsilon$-amines of one $\mathrm{Ub}$ molecule and the C-terminal carboxylic acid of another $\mathrm{Ub}$ molecule. This will lead to eight differently linked homotypic poly-Ub chains, each triggering distinct signaling events. ${ }^{2}$ All of these linkages have been detected in cells and their abundance changes during specific cellular events, indicative of their different functions. ${ }^{3}$ On top of this, different linkages can be combined in one chain resulting in so-called heterotypic or mixed-linkage chains as well as the formation of branched chains. ${ }^{4}$ Extensive investigations of Ub modifications led to the discovery of proteins that are able to specifically assemble, recognize, and break down poly-Ub chains. ${ }^{1 a}$ Recently, the development of Ub-derived activity-based probes has been instrumental in allowing us to study deubiquitinating enzymes (DUBs), enabling us to study their molecular mechanisms of action in recognizing and breaking down specific $\mathrm{Ub}$ chains. ${ }^{5}$ Significant biochemical effort has been made in finding enzyme combinations that can give access to sufficient amounts of homotypically pure poly-Ub material. ${ }^{6}$ Most of these biochemical techniques rely on the assembly of $\mathrm{Ub}$ chains by specific E2/E3 enzymes, and seven out of eight linkages can be assembled in this manner. Some of the enzyme combinations reported, however, are not $100 \%$ linkage specific, and further sample processing using DUBs is needed to remove traces of other linkages and ensure a homologous preparation. ${ }^{7}$ The
K27-linked poly-Ub chain, however, remains unattainable using biochemical approaches. Thus, extending the chemical toolbox seems important to provide new and complex Ub-derived material to further study $\mathrm{Ub}$ biology. The focus of chemical strategies to construct large polypeptides or proteins has been on the native chemical ligation (NCL) reaction, where a cysteine peptide reacts with a thioester peptide followed by trans-thiolation and S-to- $\mathrm{N}$-acyl migration giving an amide bond as final product. The NCL reaction is widely used to construct large polypeptides or proteins. This approach initially was limited to cysteine ligation sites, but after the introduction of good desulfurization strategies ${ }^{8}$ and development of thiolcontaining amino acid derivatives ${ }^{9}$ has found broader applications. The use of thiolysine peptides, for instance, opened the way to construct ubiquitin conjugates, ubiquitin dimers, and assay reagents based on $\mathrm{Ub} .{ }^{10} \mathrm{Ub}$-thioesters can be accessed in several ways ranging from intein expression to utilizing E1 enzyme activation and chemical synthesis. ${ }^{11}$ More critical is the thiolysine mutant that can be made using either GOPAL or fully synthetic strategies. ${ }^{10 a, 12} \mathrm{We}$ and other laboratories have reported syntheses toward orthogonally protected thiolysine amino acids. ${ }^{10 a, 12 b, 13}$ On the basis of such thiolysine and thioester mutants, $\mathrm{Ub}$ conjugates and small $\mathrm{Ub}$ chains have been prepared. Polymerization studies toward poly-Ub chains containing unnatural linkages have been reported using thiol-ene and copper-catalyzed click chemistries, respectively. ${ }^{14}$ The construction of di-, tri-, and tetramer $\mathrm{Ub}$ chains containing native linkages requires tedious and highly specialized synthetic strategies. ${ }^{15}$ In order to make longer Ub chains more easily accessible, we set out to develop a

Received: October 2, 2017

Published: November 27, 2017 
protocol based on a thiolysine NCL approach in order to polymerize Ub mutants in a single reaction. Not only can such an approach be applied to the synthesis of poly-Ub, but other Ub-like polymers such as, for instance, poly-SUMO could also be envisioned using similar methodology. Starting from $\gamma$ chlorolysine, ${ }^{13 \mathrm{~b}}$ we were able to obtain the orthogonally protected thiolysine derivative $\mathbf{4}$ in nine straightforward steps with $69 \%$ overall yield on a $10 \mathrm{~g}$ scale (see Scheme 1). The

Scheme 1. Synthesis of Orthogonally Protected $\boldsymbol{\gamma}$-Thiolysine

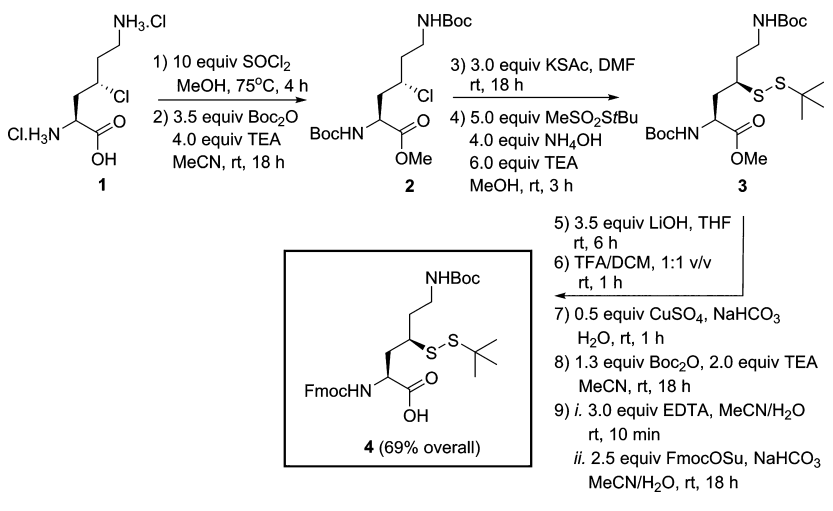

advantage of this route over previously published routes is that only one flash-column chromatography purification is needed at the end of all steps. Furthermore, the use of the 9-BBN protective group is circumvented, and only standard chemical reagents are used to protect the amino acid. Although 9-BBN served well to mask the $\alpha$-amine and carboxylic ester simultaneously, use of silica gel column chromatography as a means to remove 9-BBN following deprotection has proven difficult on larger scales. By utilizing a copper complex as protection to replace $9-\mathrm{BBN}$, deprotection, and purification are greatly simplified without affecting overall efficiency.

First, the carboxylic acid functionality of fully unprotected $\gamma$ chlorolysine $\mathrm{HCl}$ salt (1) was masked as a methyl ester, and subsequently, both amines were Boc protected to yield 2 in $98 \%$ overall yield. Substitution of the chloride using KSAc and removal of the acetyl moiety followed by introduction of the $\mathrm{StBu}$ group using S-tert-butyl methanethiosulfonate put the crucial sulfur atom in place as its orthogonally protected disulfide (3). The deprotection of the acetyl functionality and the introduction of the $\mathrm{StBu}$ disulfide were carried out in one pot, which was crucial to prevent racemization at this stage. Saponification of the ester and concomitant removal of both Boc groups was accomplished in $78 \%$ subsequent yield over four steps. The formation of a copper complex assured masking of both the $\alpha$-amine and carboxylic acid allowing for the selective Boc protection of the $\varepsilon$-amine in $97 \%$ yield. The symmetric copper complex proved highly stable, as it was readily isolated and stored without problems. Final removal of the copper atom using EDTA as alternative for more commonly used 8-hydroxyquinoline and subsequent Fmoc introduction on the $\alpha$-amine followed by the only column purification in this nine-step synthesis gave the fully orthogonal protected thiolysine derivative 4 in 93\% yield and excellent purity. With the Fmoc/Boc/StBu protection scheme in place, this amino acid could be used in Fmoc-based solid-phase peptide chemistry without the need to modify general protocols. Furthermore, this protocol provides a better overall yield, and the scalability of this method was verified in a large- scale synthesis (yielding $47.7 \mathrm{~g}$ of product 4 ). Incorporation of $\gamma$-thiolysine building block 4 at any desired position substituting the natural occurring lysines in $\mathrm{Ub}$ was achieved using our previously reported solid-phase peptide synthesis protocol, which hinges around the use of pseudoproline- and dimethoxybenzyl-dipeptide building blocks in a linear approach. $^{10 a}$ In a typical synthesis, we prepared a thiolysine mutant $\mathrm{Ub}_{1-75}$ on trityl resin (5), released it from the resin using mild acidic conditions ( $20 \mathrm{vol} \%$ hexafluoroisopropanol in dichloromethane) which liberated only the C-terminal carboxylic acid while leaving all other protective groups in place (6). Activation of the C-terminus followed by coupling to glycinyl-ethylthioester afforded 7, and global deprotection using strong acidic conditions resulted in the crude Ub mutant (8) carrying both a thiolysine and a thioester moiety (see Scheme 2). Final RP-HPLC purification followed by lyophilization of

Scheme 2. Solid Phase Peptide Synthesis of $\gamma$-Thiolysine Ubiquitin Thioester

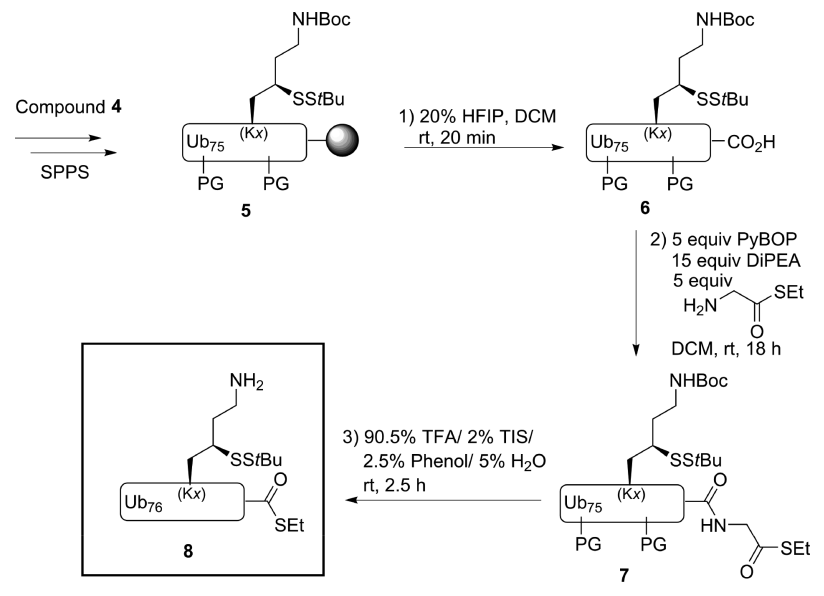

the appropriate fractions yielded the target thiolysine- $\mathrm{Ub}_{76}$ thioester as white powder in multimilligram amounts. Because the selected disulfide protective group on the thiol remains in place during acidic treatment and HPLC purification, no polymerization or cyclization side reactions occurred at this stage and the $\mathrm{Ub}$ mutants could be stored. Reactions with $\delta$ thiolysine $\mathrm{Ub}$ mutants have been reported, and polymerization was observed in the attempted syntheses for M1, K48, and K63 chains. ${ }^{16}$ An intramolecular cyclization reaction competed severely with the intermolecular polymerization reaction and led to cyclic material, and this approach was not explored further. While a $\delta$-thiolysine was employed previously, here we use a $\gamma$-thiolysine. Other differences are that we use a ethylmercaptan thioester and higher protein concentrations than reported previously. Perhaps these differences are key to a successful polymerization, although direct comparison of the $\delta$ thiolysine versus $\gamma$-thiolysine in formation of $\mathrm{diUb} \mathrm{NCL}$ experiments showed little difference. ${ }^{13 \mathrm{~b}}$ We observed superior results when using such optimized conditions; ${ }^{10 a}$ high concentrations of thiolysine-Ub-thioester reactant $(50 \mathrm{mg} /$ $\mathrm{mL}$ ) under denaturing conditions (6 M guanidinium hydrochloride/100 mM sodium phosphate buffer, $\mathrm{pH}$ 7.6) under the agency of mercaptophenylacetic acid (MPAA) as additive. Efficient formation of poly-Ub chains was found for K6, K11, K27, K29, K33, and K48 thiolysine mutants. However, the K63 mutant showed no formation of larger chains than the dimeric species (see SI Figure 3). 
To the best of our knowledge, this is the first full synthetic one-pot strategy allowing for the formation of poly-Ub chains of the K27-linkage, although recently $\mathrm{K} 27 \mathrm{Ub}_{4}$ was synthesized in a convergent approach. ${ }^{15 \mathrm{~d}} \mathrm{We}$ reasoned that the extent of polymerization could be controlled via the addition of a Ubthioester not containing the thiolysine mutation. If such a monofunctional $\mathrm{Ub}$ is ligated to the distal end of the growing $\mathrm{Ub}$ chain, polymerization would be terminated since no further thiol group is present to accommodate a following NCL reaction. It turned out that controlling speed of polymer formation and hence the length of the obtained polymers was not trivial since the NCL reaction speed varied depending on the thiolysine position in the Ub. It turned out we could use a termination reactant to decorate the most distal N-terminus of the polymer with an entity of choice such as for instance a fluorescent dye (5-carboxyrhodamine 110) or a biotin handle with relative ease (see Scheme 3). By spiking in labeled

Scheme 3. Polymerization Reaction of K33 $\gamma$-Thiolysine Ubiquitin Thioester
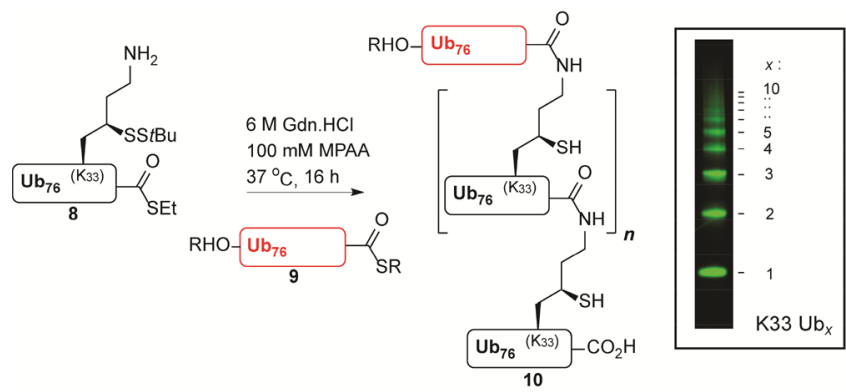

thioester (9) in the NCL reaction one incorporates the modified $\mathrm{Ub}$ molecule on the most distal $\mathrm{Ub}$ moiety of the growing chain thereby preventing further chain growth on the $\mathrm{N}$-terminal side. Spontaneous hydrolysis of the thioester moiety stops chain elongation on the C-terminus leading to the final poly-Ub chain. Intriguingly, the K29- and K33- polymerizations seemed to perform best, and the polymerization profile showed the highest degree of oligomers (over $10 \mathrm{Ub}$ units).

Dilution and dialysis of the reaction mixtures followed by cation-exchange chromatography allowed us to enrich for specific polymer length. At this stage, we were curious to see whether these fully synthetic Ub polymers could be recognized by enzymes that can cleave them, even without prior desulfurization. Initial proteolysis experiments using the panspecific DUB vOTU ${ }^{17}$ confirmed that both $\mathrm{Ub}_{2}$ and $\mathrm{Ub}_{3}$ of K29-linkage and K33-linkage were processed (see Scheme 4). Although vOTU is generally regarded as a DUB reactive toward all chains, previous studies show a diminished activity toward K29 dimers. ${ }^{18}$ This indeed is reflected in our experiments where we observe a fast turnover of both $\mathrm{K} 33$-linked $\mathrm{Ub}_{2}$ and $\mathrm{Ub}_{3}$ whereas K29-linked chains are processed less swiftly. Encouraged by these results we tested the DUB TRABID which has a preferred proteolytic activity on K29- and K33linked Ub. TRABID was also able to process both $\mathrm{Ub}_{2}$ and $\mathrm{Ub}_{3}$ species of K29- and K33-linkage, showing a faster turnover for the K29-linked polymers than the K33-linked polymers. Similar results were found previously in a FRET-based assay cleaving $\mathrm{Ub}_{2}$ by TRABID. ${ }^{10 \mathrm{c}}$ All results obtained using our fully synthetic Ub chains seem in agreement with previous reported results indicative of the success of our approach in generating
Scheme 4. Fluorescent Scan of Gels Showing Proteolysis of $\mathrm{K} 29-\mathrm{Ub}_{2}, \mathrm{~K} 33-\mathrm{Ub}_{2}$, and $\mathrm{Ub}_{3}(2 \mu \mathrm{M})$, respectively, by vOTU $(1 \mu \mathrm{M})$ or TRABID $(500 \mathrm{nM})$ Followed over Time
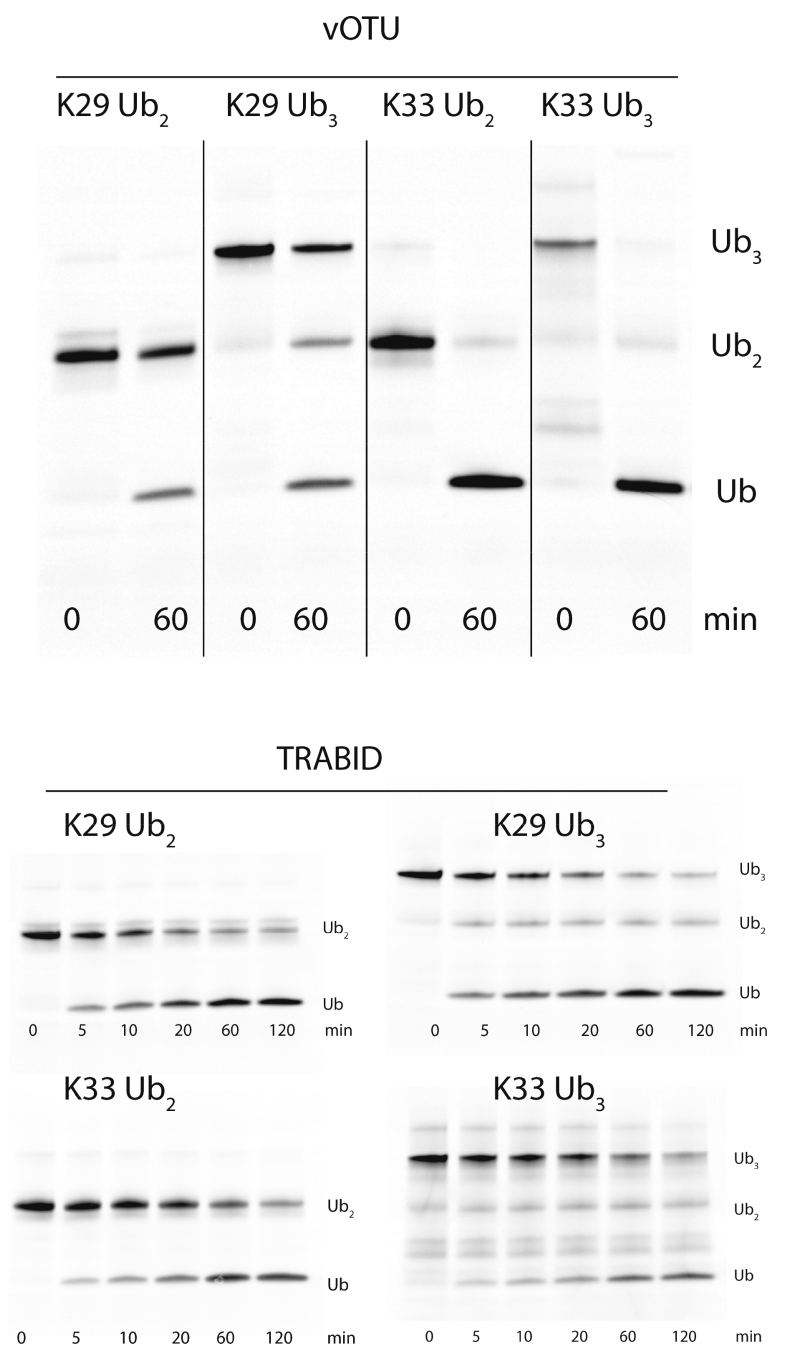

higher order $\mathrm{Ub}$ chains that fold and behave similar as material from natural origin.

In conclusion, we have shown an optimized route toward a fully orthogonal protected $\gamma$-thiolysine amino acid. This route avoids the use of the 9-BBN protective group and only requires one silica gel column purification in the entire route, and we tested scalability affording $47.7 \mathrm{~g}$ of final product 4 . This modified amino acid can be readily incorporated into $\mathrm{Ub}$ mutants using standard solid phase peptide chemistry, leading to high purity synthetic proteins. Such synthons can be used in a one-pot polymerization approach giving quick access to large poly-Ub chains of well-defined linkage. Validation of recognition and processing of these materials by highly specific proteases reveals that this method is a valuable tool in the construction of $\mathrm{Ub}$ derived polymers.

\section{ASSOCIATED CONTENT}

\section{Supporting Information}

The Supporting Information is available free of charge on the ACS Publications website at DOI: 10.1021/acs.orglett.7b03085.

Synthetic protocols and characterization of crucial intermediates (PDF) 


\section{AUTHOR INFORMATION}

\section{Corresponding Authors}

*E-mail: gvanderheden@lumc.nl.

*E-mail: h.ovaa@lumc.nl.

ORCID

Gerbrand J. van der Heden van Noort: 0000-0001-5955-6431

Author Contributions

${ }^{\S}$ G.J.vdHvN. and R.K. contributed equally.

Notes

The authors declare no competing financial interest.

\section{ACKNOWLEDGMENTS}

We thank Dris el Atmioui and Cami Talavera Ormeňo (Department of Chemical Immunology, LUMC, Leiden) for SPPS. This work was supported by an NWO VENI grant to G.J.vdHvN. and an ERC grant to H.O.

\section{REFERENCES}

(1) (a) Komander, D.; Rape, M. Annu. Rev. Biochem. 2012, 81, 203229. (b) Hochstrasser, M. Nature 2009, 458, 422-429.

(2) Komander, D. Biochem. Soc. Trans. 2009, 37, 937.

(3) Xu, P.; Duong, D. M.; Seyfried, N. T.; Cheng, D.; Xie, Y.; Robert, J.; Rush, J.; Hochstrasser, M.; Finley, D.; Peng, J. Cell 2009, 137, 133145 .

(4) Kim, H. T.; Kim, K. P.; Lledias, F.; Kisselev, A. F.; Scaglione, K. M.; Skowyra, D.; Gygi, S. P.; Goldberg, A. L. J. Biol. Chem. 2007, 282, $17375-17386$.

(5) (a) Mulder, M. P. C.; El Oualid, F.; ter Beek, J.; Ovaa, H. ChemBioChem 2014, 15, 946-949. (b) Ekkebus, R; van Kasteren, S. I.; Kulathu, Y.; Scholten, A.; Berlin, I.; Geurink, P. P.; de Jong, A.; Goerdayal, S.; Neefjes, J.; Heck, A. J. R.; Komander, D.; Ovaa, H. J. Am. Chem. Soc. 2013, 135, 2867-287. (c) Flierman, D.; van der Heden van Noort, G. J.; Ekkebus, R.; Geurink, P. P.; Mevissen, T. E. T.; Hospenthal, M. K.; Komander, D.; Ovaa, H. Cell Chem. Biol. 2016, 23, 472-482.

(6) Faggiano, S.; Alfano, C.; Pastore, A. Anal. Biochem. 2016, 492, $82-90$.

(7) (a) Michel, M. A.; Elliott, P. R.; Swatek, K. N.; Simicek, M.; Pruneda, J. N.; Wagstaff, J. L.; Freund, S. M. V.; Komander, D. Mol. Cell 2015, 58, 95-109. (b) Kristariyanto, Y. A.; Abdul Rehman, S. A.; Campbell, D. G.; Morrice, N. A.; Johnson, C.; Toth, R.; Kulathu, Y. Mol. Cell 2015, 58, 83-94.

(8) Wan, Q.; Danishefsky, S. J. Angew. Chem. Int. Ed. 2007, 46, 9248-9252.

(9) (a) Haase, C.; Rohde, H.; Seitz, O. Angew. Chem. Int. Ed. 2008, 47, 6807-6810. (b) Crich, D.; Banerjee, A. J. Am. Chem. Soc. 2007, 129, 10064-1006. (c) Shang, S.; Tan, Z.; Dong, S.; Danishefsky, S. J. J. Am. Chem. Soc. 2011, 133, 10784-10786. (d) Harpaz, Z.; Siman, P.; Kumar, K. S. A.; Brik, A. ChemBioChem 2010, 11, 1232-1235. (e) Chen, J.; Wang, P.; Zhu, J.; Wan, Q.; Danishefsky, S. J. Tetrahedron 2010, 66, 2277-2283.

(10) (a) El Oualid, F.; Merkx, R.; Ekkebus, R.; Hameed, D. S.; Smit, J. J.; de Jong, A.; Hilkmann, H.; Sixma, T. K.; Ovaa, H. Angew. Chem. Int. Ed. 2010, 49, 10149-10153. (b) Geurink, P. P.; El Oualid, F.; Jonker, A.; Hameed, D. S.; Ovaa, H. ChemBioChem 2012, 13, 293297. (c) Geurink, P. P.; van Tol, B. D. M.; van Dalen, D.; Brundel, P. J. G.; Mevissen, T. E. T.; Pruneda, J. N.; Elliott, P. R.; van Tilburg, G. B. A.; Komander, D.; Ovaa, H. ChemBioChem 2016, 17, 816-820

(11) (a) Burchak, O. N.; Jaquinod, M.; Cottin, C.; Mugherli, L.; Iwai, K.; Chatelain, F.; Balakirev, M. Y. ChemBioChem 2006, 7, 1667-1669.

(b) Borodovsky, A.; Ovaa, H.; Kolli, N.; Gan-Erdene, T.; Wilkinson, K. D.; Ploegh, H. L.; Kessler, B. M. Chem. Biol. 2002, 9, 1149-1159.

(12) (a) Virdee, S.; Kapadnis, P. B.; Elliott, T.; Lang, K.; Madrzak, J.; Nguyen, D. P.; Riechmann, L.; Chin, J. W. J. Am. Chem. Soc. 2011, 133, 10708-10711. (b) Ajish Kumar, K. S.; Haj-Yahya, M.;
Olschewski, D.; Lashuel, H. A.; Brik, A. Angew. Chem. Int. Ed. 2009, 48, 8090-8094.

(13) (a) Pasunooti, K. K.; Yang, R.; Vedachalam, S.; Gorityala, B. K.; Liu, C.-F.; Liu, X.-W. Bioorg. Med. Chem. Lett. 2009, 19, 6268-6271. (b) Merkx, R.; de Bruin, G.; Kruithof, A.; van den Bergh, T.; Snip, E.; Lutz, M.; El Oualid, F.; Ovaa, H. Chem. Sci. 2013, 4, 4494-4498.

(14) (a) Schneider, T.; Schneider, D.; Rösner, D.; Malhotra, S.; Mortensen, F.; Mayer, T. U.; Scheffner, M.; Marx, A. Angew. Chem. Int. Ed. 2014, 53, 12925-12929. (b) Trang, V. H.; Valkevich, E. M.; Minami, S.; Chen, Y.-C.; Ge, Y.; Strieter, E. R. Angew. Chem. Int. Ed. 2012, 51, 13085-13088.

(15) (a) Kumar, K. S. A.; Bavikar, S. N.; Spasser, L.; Moyal, T.; Ohayon, S.; Brik, A. Angew. Chem., Int. Ed. 2011, 50, 6137-614. (b) Bekes, M.; van der Heden van Noort, G. J.; Ekkebus, R.; Ovaa, H.; Huang, T. T.; Lima, C. D. Mol. Cell 2016, 62, 572-58. (c) Pan, M.; Gao, S.; Zheng, Y.; Tan, X.; Lan, H.; Tan, X.; Sun, D.; Lu, L.; Wang, T.; Zheng, Q.; Huang, Y.; Wang, J.; Liu, L. J. Am. Chem. Soc. 2016, 138, 7429-7435. (d) Liu, L.; Tang, S.; Liang, L.-J.; Si, Y.-Y.; Gao, S.; Wang, J.; Liang, J.; Mei, Z.; Zheng, J. Angew. Chem., Int. Ed. 2017, 56, 13333-13337.

(16) Moyal, T.; Bavikar, S. N.; Karthikeyan, S. V.; Hemantha, H. P.; Brik, A. J. Am. Chem. Soc. 2012, 134, 16085-16092.

(17) Hospenthal, M. K.; Mevissen, T. E. T.; Komander, D. Nat. Protoc. 2015, 10, 349-361.

(18) Akutsu, M.; Ye, Y.; Virdee, S.; Chin, J. W.; Komander, D. Proc. Natl. Acad. Sci. U. S. A. 2011, 108 (6), 2228-2233. 